\title{
Producción de artículos primarios relacionados con la inteligencia emocional en estudiantes ecuatorianos
}

Production of primary articles related to emotional intelligence in Ecuadorian students

Produção de artigos primários relacionados à inteligência emocional em estudantes equatorianos

\section{J. Roberto Terán'}

jroberto-teran@hotmail.com

https://orcid.org/0000-0002-4142-4120
Exequiel Guevara2,3

exequiel.gd@gmail.com

https://orcid.org/0000-0001-7250-7319

${ }^{1}$ Instituto Tecnológico Universitario Cordillera. Quito, Ecuador

${ }^{2}$ Centro de Capacitación e Investigación en Neurociencias (CINEURO). Temuco, Chile

${ }^{3}$ Departamento de Investigación y Desarrollo Educativo (DIDE). Corporación Educacional Niños Felices. Vilcún, Chile

Recibido: junio 2021 / Revisado: julio 2021 / Aceptado: agosto 2021 / Publicado: septiembre 2021

\section{INTRODUCCIÓN}

El concepto de Inteligencia Emocional (IE) fue popularizado por Daniel Goleman en el año 1995, quien, a pesar de que sus aseveraciones no han sido científicamente demostradas, fue probablemente el responsable de que el término se conociera por el público general hasta la actualidad (Extremera, et. al, 2003).

Para Mayer y Salovey (1997), el concepto de IE se considera como la capacidad para procesar la información emocional, quienes, además, fueron los primeros en acuñar este término ya en los inicios de la década del 90 (Salovey, 1990).

Si bien existen algunos programas basados en IE con resultados positivos para intervenir en estudiantes (Fernández, 2005), en Ecuador aún su estudio y aplicación son incipientes.

\section{METODOLOGÍA}

Se realizó una búsqueda en las bases de datos de libre acceso Pubmed, Scielo, Redalyc y Dialnet, utilizando los siguientes términos y operadores booleanos tanto para español: ((Inteligencia emocional) AND (estudiantes OR universitarios)AND (Ecuador ORecuatorianos)); como para inglés: ((emotional intelligence) AND (students OR college student) AND (Ecuador OR Ecuadorian)). La búsqueda inicial generó un total de 27 artículos científicos, de los cuales, al realizar un filtro por medio del análisis del título, resumen, pertinencia del tema y los reduplicados (uno), sólo cinco fueron seleccionados (Tabla 1 ). 
Tabla 1. Lista con los artículos seleccionados.

\begin{tabular}{|c|c|c|c|}
\hline $\begin{array}{l}\text { Año de } \\
\text { publicación }\end{array}$ & Autores & Nombre del artículo & $\begin{array}{c}\text { Base de } \\
\text { datos }\end{array}$ \\
\hline 2017 & Taramuel J, Zapata V. & $\begin{array}{l}\text { Aplicación del test TMMS-24 para el análisis y } \\
\text { descripción de la Inteligencia Emocional considerando } \\
\text { la influencia del sexo. }\end{array}$ & Dialnet \\
\hline 2018 & $\begin{array}{l}\text { Loor-Rivadeneira RM, } \\
\text { Durán-Solórzano S, } \\
\text { Guerrero-Saltos DR. }\end{array}$ & $\begin{array}{l}\text { Inteligencia emocional y su relación con la } \\
\text { autoeficacia generalizada en estudiantes de la } \\
\text { escuela de Psicología de la Universidad Técnica de } \\
\text { Manabí, Portoviejo, Ecuador. }\end{array}$ & Dialnet \\
\hline 2019 & $\begin{array}{l}\text { Vega J, Bello Z, } \\
\text { Medina J, } \\
\text { Alfonso A. }\end{array}$ & $\begin{array}{l}\text { Un estudio de las competencias emocionales en } \\
\text { estudiantes de Ciencias de la Salud de la Universidad } \\
\text { Técnica de Manabí. }\end{array}$ & Dialnet \\
\hline 2020 & Quishpe-Hipo L. & Inteligencia Emocional en el aula de inglés & Dialnet \\
\hline 2021 & $\begin{array}{l}\text { Cañas-Lerma A, } \\
\text { Cuartero-Castañer M, } \\
\quad \text { Mascialino G, } \\
\text { Hildaldo-Andrade P. }\end{array}$ & $\begin{array}{l}\text { Empathy and COVID-19: Study in Professionals and } \\
\text { Students of the Social Health Field in Ecuador. }\end{array}$ & Pubmed \\
\hline
\end{tabular}

\section{A MANERA DE CIERRE}

Deacuerdo a lo demostrado previamente, la investigación en IE en estudiantes ecuatorianos, de cualquier nivel, aún es incipiente y escasa, existiendo evidencia publicada por medio de artículos científicos primarios únicamente en los últimos 5 años. Asimismo, los estudios se enfocan principalmente sólo en caracterizar a los participantes en relación a la IE, existiendo un nulo trabajo desde el punto de vista experimental o cuasi-experimental.

Finalmente, se espera que los resultados emanados de la presente investigación, sirvan de aliciente para alentar tanto a investigadores como a docentes y profesionales de distintas índoles, a seguir generando evidencia en este interesante tópico.

\section{REFERENCIAS}

Cañas-Lerma A, Cuartero-Castañer $M$, Mascialino G, Hildaldo-Andrade P. (2021) Empathy and COVID-19: Study in Professionals and Students of the Social Health Field in Ecuador. Int. J. Environ. Res. Public Health. 18(338):1-9

Extremera N, Fernández-Bérrocal P (2003). La inteligencia emocional en el contexto educativo: hallazgos científicos de sus efectos en el aula. Revista de Educación. 332:97-116

Fernández P, Extremera N. (2005) La Inteligencia Emocional y la educación de las emociones desde el Modelo de Mayer 
y Salovey. Revista Interuniversitaria de Formación del Profesorado.; 19(3):63-93

Loor-Rivadeneira RM, Durán-Solórzano S, Guerrero-Saltos DR. (2018) Inteligencia emocional y su relación con la autoeficacia generalizada en estudiantes de la escuela de Psicología de la Universidad Técnica de Manabí, Portoviejo, Ecuador. Polo del Conocimiento. 28(3):284-297

Mayer JD, Salovey P. (1997) What is emotional intelligence? En Salovey P, Sluyter D, (Eds.). Emotional development and emotional intelligence: Implications for educators. Basic Books; p. 3-31

Quishpe-Hipo, L. (2020). Inteligencia Emocional en el aula de inglés. Dominio de las Ciencias. 6(2):319-339

Salovey P, Mater JD. (1990) Emotional Intelligence. Imagination, Cognition, and Personality. 9:185-211
Taramuel J, Zapata V. Aplicación del test TMMS-24 para el análisis y descripción de la Inteligencia Emocional considerando la influencia del sexo. (2017) Revista Publicando. 4(11):162-181.

Vega J, Bello Z, Medina J, Alfonso A. (2019) Un estudio de las competencias emocionales en estudiantes de Ciencias de la Salud de la Universidad Técnica de Manabí. Revista SINAPSIS; 14(1):1-14

Conflicto de intereses: Ninguno declarado por los autores.

Financiación: Ninguna declarada por los autores.

Agradecimiento: Ninguno manifestado por los autores 